\title{
Care that ran smoothly, was responsive to clients' needs, and required no special effort for clients to maintain was important for continuity in home care
}

Woodward CA, Abelson J, Tedford S, et al. What is important to continuity in home care? Perspectives of key stakeholders. Soc Sci Med 2004;58:177-92.

\section{Q What are key stakeholders' (clients, caregivers, case managers, and home care workers) perspectives of continuity in home care?}

DESIGN

Qualitative study.

\section{SETTING}

Hamilton, Ontario, Canada.

\section{PARTICIPANTS}

13 case managers, 19 home service providers, 25 clients, 5 caregivers, and 3 physicians who had patients receiving home care. All participants were linked to a community care access centre.

\section{METHODS}

Data were collected mainly by indepth telephone interviews, although some interviews were done face to face. Interviews lasted 45-90 minutes. All but 3 interviews were tape recorded and transcribed (interviews not tape recorded were recorded by hand written notes and dictation after the interviews).

An iterative process of data collection and analysis was used to confirm and reconfirm emerging themes. 4 researchers read text associated with various codes and triangulated their interpretations to develop higher level meaning. Data were analysed using a coding scheme derived from preliminary analysis. Input from an advisory group was sought to ensure accuracy of the findings.

\section{MAIN FINDINGS}

Continuity of care was experienced as care that ran smoothly, was responsive to clients' needs, and required no special effort for clients to maintain. 2 dimensions of care emerged as important to facilitating continuity. Managing care referred to the importance of care planning, monitoring, and regular review by service providers and case managers, and the importance of care coordination (ensuring the care process was managed, and development and communication of the care plan and care delivery process). Service provision highlighted the importance of continuous service delivery and prevention of interrupted service; consistent, appropriate knowledge and skills (ie, knowledge of the client, the care required, and the home); ongoing, accurate observation by providers so that changes in a client's condition would be monitored; trusting relationships between service providers and clients and caregivers; rapport among care team members to ensure positive and effective working relationships; and consistent timing of care for each day. Communication and consistent personnel support were important to providing continuity in home care. Clients emphasised consistency of timing of service delivery as important attributes for providing continuity of care, and service providers and case managers emphasised care management issues (eg, care planning, monitoring, and coordination). Service providers and case managers identified

For correspondence: $\operatorname{Dr} \mathrm{C}$ A Woodward, Department of Clinical Epidemiology and Biostatistics, McMaster University, Hamilton, Ontario, Canada. woodward@mcmaster.ca

Sources of funding: Canadian Health Services Research Foundation and Ontario Ministry of Health and Long Term Care. several groups of clients requiring high levels of continuity of care (ie, people with chronic health problems that involve physical, mental health, or cognitive disabilities; people in the terminal phases of illness; very old people; people with complex medical regimens; and people whose caregivers are under considerable stress).

\section{CONCLUSIONS}

Key stakeholders' (clients, caregivers, case managers, and home care workers) described continuity in home care as care that ran smoothly, was responsive to clients' needs, and required no special effort for clients to maintain. Managing care and service provision were 2 dimensions of care that were important for facilitating continuity of home care.

\section{Commentary}

n Canada, unprecedented attention on home care from governments and decision makers has created opportunities to optimise the potential of home care within the healthcare system. ${ }^{\prime}$ Research to direct this evolution in home care is paramount at this time. The study by Woodward et al contributes key perspectives of how continuity of care in home care is conceptualised by patients, their families, and providers.

The qualitative approach was an appropriate method to carefully and systematically show the components of continuity in home care, adding important understanding of a concept yet to be clearly defined in the literature. ${ }^{2}$ However, further research will be needed to establish the relation between the 2 dimensions of care and the components experienced as continuity in home care that were identified in this study. From this research, base indicators of continuity can be developed to evaluate home care services, an important objective identified by the researchers.

With increasingly complex practice environments and care issues, greater emphasis on efficient, effective information and communication systems to support home care services is needed. The study findings emphasise that, although these are key to continuity, communication systems cannot be a substitute for the relationships and trust developed through consistent personnel. Furthermore, the importance of continuity for home care practice is accentuated by the finding that the types of clients identified as requiring a high level of continuity include most of the client population served by home care. As a result, the study findings have meaning for both home care practitioners and administrators, reinforcing the rationale for ensuring that continuity in care at home is maximised when changes to care processes and service delivery models are implemented.

Della K Roberts, MSN, CNS Hospice Palliative Care Delta, Fraser Health Authority Delta, British Columbia, Canada

1 Sharkey S, Larsen L, Mildon B. An overview of home care in Canada: its past, present, and potential. Home Health Care Management \& Practice 2003;15:382-90.

2 Sparbel KJ, Anderson MA. Integrated literature review of continuity of care: Part 1, conceptual issues. J Nurs Scholarsh 2000;32:17-24. 